\title{
Experimental Data for Study on the Shielding Effect of Electromagnetic Wave
}

\author{
Chen-Ching Ting, ${ }^{1, *}$, Chien-Chih Chen ${ }^{2}$ \\ ${ }^{1}$ Department of Mechanical Engineering, National Taipei \\ University of Technology, Chinese Taipei \\ ${ }^{2}$ Institute of Mechanical and Electrical Engineering, National Taipei \\ University of Technology, Chinese Taipei \\ E-mail: chchting@ntut.edu.tw, s3300357@ntut.edu.tw \\ Received November 16, 2010, revised June 13, 2011; accepted June 25, 2011
}

\begin{abstract}
This article presents some new experimental data for study the shielding effect of electromagnetic wave. The frequencies of electromagnetic wave are mainly focused on emission of daily electrical equipments, which are normally called "low frequency electromagnetic waves". In this work, a water pump with maximum magnetic field intensity of ca. $2300 \mathrm{mG}$ was applied as emission source of the electromagnetic wave. Experimental measurements used various shielding materials with the major constituent iron $(\mathrm{Fe})$ in the form of plate for studying shielding effect of electromagnetic wave. The studied parameters were different thicknesses and gaps of the plate. The results show that pure iron plate has the best effect for shielding the magnetic field and its transmission ratio of magnetic field is proportional to distance between the emission source and the shielding plate. Moreover, the shielding plate close to the emission source received better protection.
\end{abstract}

Keywords: Shielding Effect, Electromagnetic Wave, Low Frequency, Iron

\section{Introduction}

It's well known that the electromagnetic waves are generated by the alternating current $(\mathrm{AC})$ due to the electromagnetic induction [1]. An electromagnetic wave consists of the mutual perpendicular electric and magnetic fields simultaneously. Figure 1 shows schematic description of the electromagnetic wave, where $\boldsymbol{E}$ is the electric field vector, $\boldsymbol{H}$ is the magnetic field vector, $\boldsymbol{P}$ is the integrated electromagnetic field vector, and $\lambda$ is wave length of the electromagnetic wave.

Since 1831 M. Faraday developed the electromagnetism and J. C. Maxwell derived the Maxwell's equations in 1875 show the phenomenon of electromagnetic induction. In recent years, the electro-communication and the electrical appliance are widely used in the world, which causes serious problem of the electromagnetic interference (EMI). The electromagnetic waves emitted by the daily electrical equipments are called "low frequency electromagnetic waves", their frequencies are normally defined as $30 \sim 300 \mathrm{~Hz}$. The sources of low frequency electromagnetic wave can be e.g. AC conducting wire, cell phone, computer, microwave, television, and so on.
The EMI often makes electrical devices out of order, i.e. error of the signal, the sound, the image, etc.

EMI on electronic devices is already known, but the same research on the biological bodies is very few due to its too short observation time. The most scientists believe that the electromagnetic radiation will hurt the bodies for a long exposure time and warn people to avoid long exposure time on the extraordinary strong electromagnetic field. The up to now understood injury by EMI on the bodies are the heating damage, the body balance destroy, and the accumulation effect. The extraordinary strong electromagnetic field inside the body will cause the water molecules to be heated while the water occupies ca. $70 \%$ of the body. Moreover, the steady situation of the body will be destroyed by the extraordinary strong electromagnetic field. In addition, the accumulation of several weak electromagnetic influences will also hurt the body.

Due to the extreme dependence on the daily electrical equipments today, people can not live without electromagnetic field. Shielding of the electromagnetic radiation is the most simple, direct, and available way to protect the EMI for reducing the surrounding quantity of 


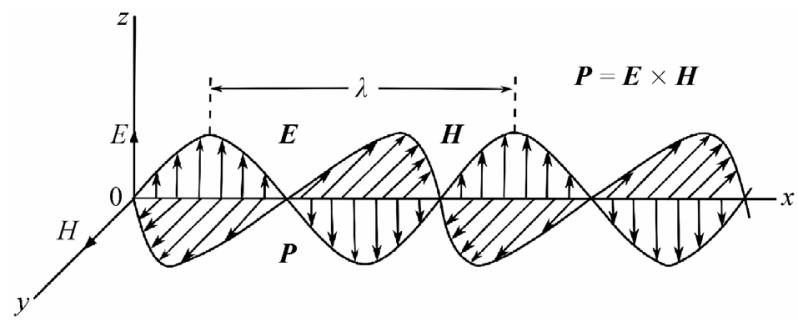

Figure 1. Schematic description of the electromagnetic wave.

electromagnetic radiation, which has been studied in many literatures [2-10]. The principle of the electromagnetic radiation shielding is to reduce the electromagnetic energy through the absorption or reflection of the materials. According to this reason, this work offers some significant experimental data for research on the shielding effect to protect the low frequency electromagnetic radiation, which is normally produced by the transformer. The applied physical principles are the skin effect and the magnetic protection effect. The skin effect is the tendency of an alternating electric current to distribute itself within a conductor so that the current density near the surface of the conductor is greater than that at its core. That is, the electric current tends to flow at the "skin" of the conductor. The skin effect causes the effective resistance of the conductor to increase with the frequency of the current. The magnetic protection effect occurs on the material including iron for protection of the alternating magnetic waves [11]. The research will use the cutting off technique to protect the electromagnetic radiation. The technique will consider type and thickness of the protecting material for measurement and analysis of transmission ratio to select the best protecting material.

\section{Basic Theory}

The electromagnetic waves are generally generated by the alternating current (AC) in terms of the Faraday's induction law and the Ampère's circuital law. The Faraday's induction law indicates that a time-varying magnetic field will have an electric field associated with it as shown in Equation (1). Moreover, the Ampère's circuital law illustrates that a time-varying electric field will be accompanied by a magnetic field as shown in Equation (2) [12]. That is, the electric and magnetic waves always exist simultaneously.

$$
\begin{gathered}
\int_{C} \boldsymbol{E} \cdot \mathrm{d} \boldsymbol{I}=-\iint_{A} \frac{\partial \boldsymbol{B}}{\partial t} \cdot \mathrm{d} \boldsymbol{S}, \\
\int_{C} \boldsymbol{B} \cdot \mathrm{d} \boldsymbol{l}=\mu \iint_{A}\left(\boldsymbol{J}+\varepsilon \frac{\partial \boldsymbol{E}}{\partial t}\right) \cdot \mathrm{d} \boldsymbol{S},
\end{gathered}
$$

where $\boldsymbol{E}$ and $\boldsymbol{B}$ are the electric and magnetic fields; $\mathrm{d} \boldsymbol{I}$ and $\mathrm{d} \boldsymbol{S}$ are differential path and traversing area of the electric and magnetic fields; $\mu, \boldsymbol{J}$, and $\varepsilon$ are permeability, current density, and electric permittivity respectively.

In principle, a conductor can stop electromagnetic wave to penetrate in terms of the skin effect. The skin effect illustrates that the electric charges within the conductor are gathered on the surface while an electromagnetic wave is propagating into it. That is, the largest current density appears at skin of the conductor and is rapidly decreased with depth of the conductor. The average depth is called as the skin depth which strongly depends on frequency of the incident electromagnetic wave. In general, the larger the frequency of the incident electromagnetic wave, the smaller the skin depth. The common relationship is

$$
J=J_{S} e^{-\mathrm{d} / \delta},
$$

where d and $\delta$ are distances of current to surface and skin depth; $J$ and $J_{S}$ are current density and surface current density in the conductor respectively. In normal cases the skin depth is well approximated as

$$
\delta=\sqrt{\frac{2 \rho}{\omega \mu}}=\sqrt{\frac{2 \rho}{(2 \pi v)\left(\mu_{0} \mu_{r}\right)}} \approx 503 \sqrt{\frac{\rho}{v \mu_{r}}}(\mathrm{~m}),
$$

where $\rho$ is resistivity of the conductor; $\omega=2 \pi \nu$ is angular frequency $(\omega)$ and frequency $(v)$ of current; $\mu, \mu_{0}$, and $\mu_{r}$ are individually absolute, free space, and relative magnetic permeability of the conductor. Figure 2 shows damping curves of electric and magnetic waves for the electromagnetic wave in the conductor [11]. Figure 2 clearly indicates that the electromagnetic wave is rapidly damped with depth in the conductor as the near hyperbolic curves.

The shielding effect of electromagnetic wave normally

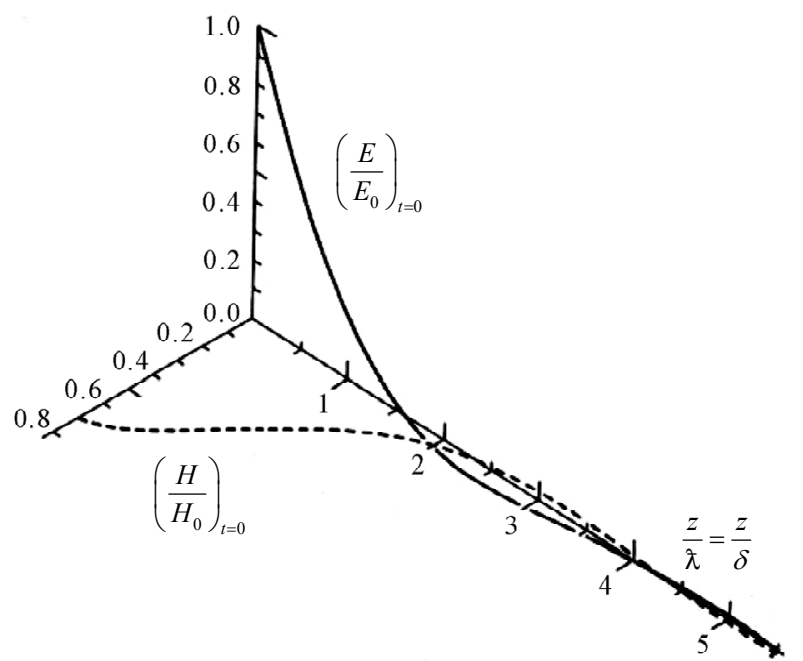

Figure 2. Damping curves of electric and magnetic waves for the electromagnetic wave in the conductor [11]. 
uses shielding materials to reduce penetration of the electromagnetic wave. In general, the skin depth in the conductor does not only depend on frequency of the incident electromagnetic wave, but also be influenced by the shielding materials. Table 1 shows some common conductive materials in corresponding to frequency of the incident electromagnetic wave and its skin depth respectively [11].

\section{Experimental Setup}

Experimental setup is to measure the decay rate and the transmission ratio of the electromagnetic wave using various shielding materials with the major constituent iron $(\mathrm{Fe})$ in the form of plate. The studied parameters are different thicknesses and gaps of the plate. The triaxial extremely low frequency (ELF) magnetic field meter (TES-1394) from TES Electrical Electronic Corp. with \pm $3 \%$ measuring error and $30 \mathrm{~Hz} \sim 2 \mathrm{kHz}$ measuring bandwidth of frequencies is used for detecting the magnetic field intensity. Table 2 exhibits the applied shielding materials and their measuring conditions, where the gap is the distance between two plates and its corresponding plate thickness is $1 \mathrm{~mm}$.

The experimental setup consists of three different integrations, the plate thickness, the plate gap, and the magnetic loop effect, to study influence of the shielding materials. Measurements are focused on the decay rate and the transmission ratio of the electromagnetic wave. The transmission ratio is defined as ratio of the penetrated magnetic field intensity and the magnetic field intensity with protection at the same position.

$$
\text { Transmission }[\%]=\frac{\text { Penetrated }}{\text { No Protection }} 100 \%
$$

Figure 3 shows graphical descriptions for measuring the decay rate and the transmission ratio with various materials and different thicknesses, where Figure 3(a) shows schema of the experimental setup and Figure 3(b) is the photo. To reduce the measuring error from the side electromagnetic field, the $50 \mathrm{~cm} \times 50 \mathrm{~cm}$ relatively large plates are used.

The left side in Figure 3(b) shows the working water pump, which was used as the emission source. The right side is the TES-1394 magnetic field meter, which was put on the opposite positions from the shielding plate. Measurements with various shielding materials simply change the intercalary plate. The decay rate measurements moved the TES-1394 magnetic field meter far away from the plate in comparison with the emission source moving far away from the plate. The performed distances were from 0 to $100 \mathrm{~cm}$ with moving step of 5 $\mathrm{cm}$.
Table 1. Some common conductive materials in corresponding to frequency of the incident electromagnetic wave and its skin depth respectively [11].

\begin{tabular}{lcccc}
\hline $\begin{array}{c}\text { Frequency } \\
\text { Skin Depth }\end{array}$ & $\begin{array}{c}60[\mathrm{~Hz}] \\
(\mathrm{cm})\end{array}$ & $\begin{array}{c}1[\mathrm{kHz}] \\
(\mathrm{mm})\end{array}$ & $\begin{array}{c}2[\mathrm{MHz}] \\
(\mathrm{mm})\end{array}$ & $\begin{array}{c}1[\mathrm{GHz}] \\
(\mu \mathrm{m})\end{array}$ \\
\hline Aluminum & 1.1 & 2.7 & 0.085 & 1.6 \\
Copper & 0.85 & 2.1 & 0.066 & 1.2 \\
Silver & 0.83 & 2.03 & 0.064 & 1.2 \\
Nickel & 0.18 & 4.4 & 0.014 & 0.26 \\
Mumetal & 0.037 & 0.092 & 0.0029 & 0.053 \\
(75Ni, 2Cr, 5Cu, 18Fe) & & & & \\
Tin & 2.21 & 5.41 & 0.171 & 3.12 \\
Zinc & 1.51 & 3.7 & 0.117 & 2.14 \\
Magnetic Iron & 0.14 & 0.35 & 0.011 & 0.2 \\
\hline
\end{tabular}

Table 2. Shielding plates with plate area of $50 \mathrm{~cm} \times 50 \mathrm{~cm}$ and their measuring conditions.

\begin{tabular}{ccc}
\hline Material & Thickness $(\mathrm{mm})$ & Gap $(\mathrm{cm})$ \\
\hline Ni-Cr Stainless & 1 & \\
Galvanized Iron & $1,2,5$ & $0,3,5$ \\
Iron & $1,2,5$ & $0,3,5$ \\
\hline
\end{tabular}

Figure 4 shows graphical descriptions for measuring the decay rate and the transmission ratio with various materials as well as different plate gaps, where Figure 4(a) shows schema of the experimental setup and Figure 4(b) is the photo. The plates with $1 \mathrm{~mm}$ thickness were used for measuring and discussing influence of the plate gaps as well as the measured plate gaps are 0,3 , and 5 cm (see Table 2).

Influence of the loop effect for transmission ratio of magnetic field inside the materials is also considered in this work. Figure 5 shows photo of the experimental setup for measuring the loop effect. Figure 5 shows that a closed circle square plate box with $L \times W \times H=50 \mathrm{~cm}$ $\times 50 \mathrm{~cm} \times 50 \mathrm{~cm}$ is built with galvanized iron. The emission source of the water pump was put into the box and moved the outside TES-1394 magnetic field meter far away from the box for measuring.

\section{Results and Discussion}

Experimental results and discussion are focused on the decay rate and the transmission ratio of magnetic field intensity using different shielding materials. Figure 6 shows relationship between the penetrated electromagnetic field intensity and the measured distances with 


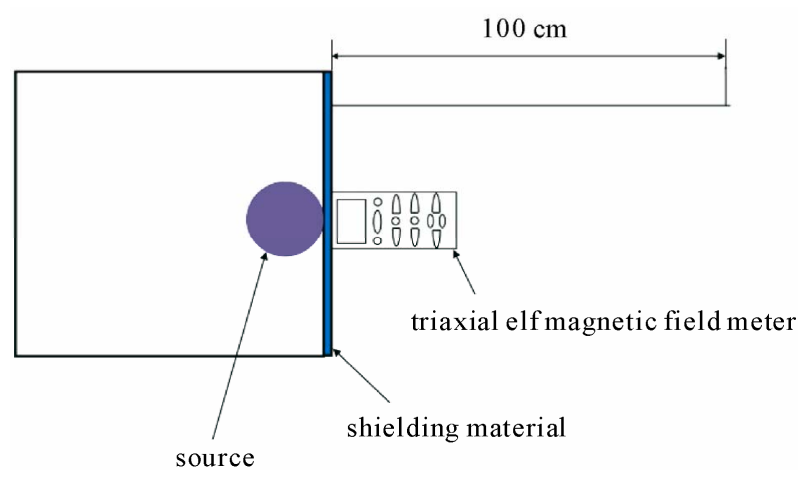

(a)

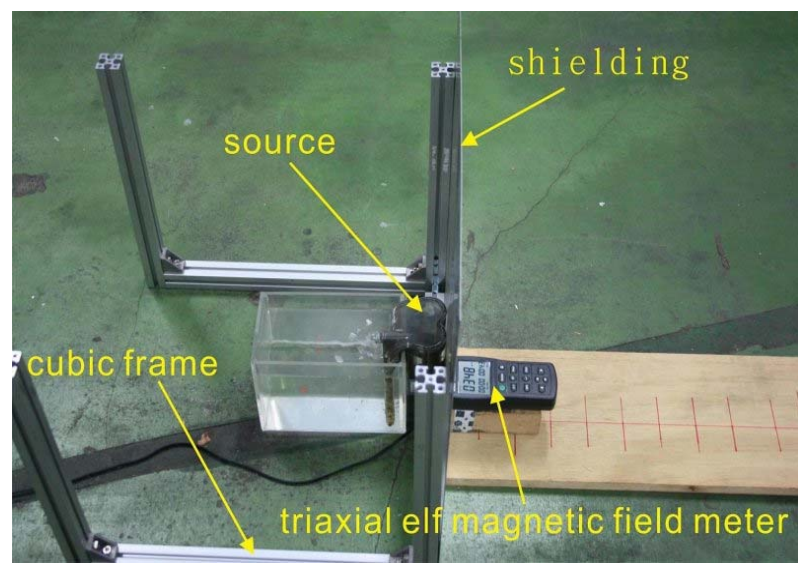

(b)

Figure 3. Graphical descriptions for measuring the decay rate and the transmission ratio with the various materials and different thicknesses. (a) Schema of the experimental setup; (b) Photo of the experimental setup.

various shielding materials, where all plate thicknesses are $1 \mathrm{~mm}$. Figure 6 clearly indicates that the shielding effect of iron plate is better than galvanized iron and $\mathrm{Ni}-\mathrm{Cr}$ stainless steel plates. The decay distance of the magnetic field intensity in Figure 6 for the four curves with ca. the same value of $30 \mathrm{~cm}$, which shows good agreement with the theory [11]. The decay distance of the magnetic field intensity is independent on the magnetic field intensity.

Figure 7 further shows relationship between the penetrated magnetic field intensity and the measured distances with different plate thicknesses of the iron. The applied plate thicknesses are 1, 2, and $5 \mathrm{~mm}$. Figure 7 exhibits that the larger the thickness of shielding plate, the better the protection of the electromagnetic field intensity. The decay distances of the three curves are ca. the same value of $30 \mathrm{~cm}$.

Figure 8 shows comparison of the galvanized iron and the iron protection. The result exhibits that protection using the iron plate is better than the galvanized iron plate.

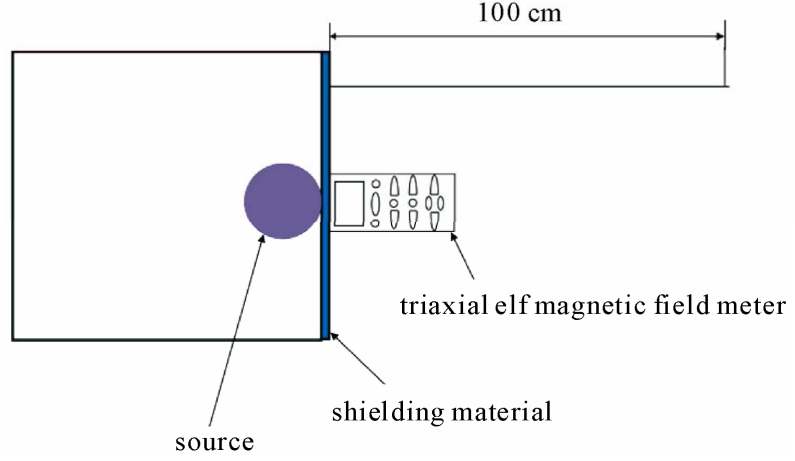

(a)

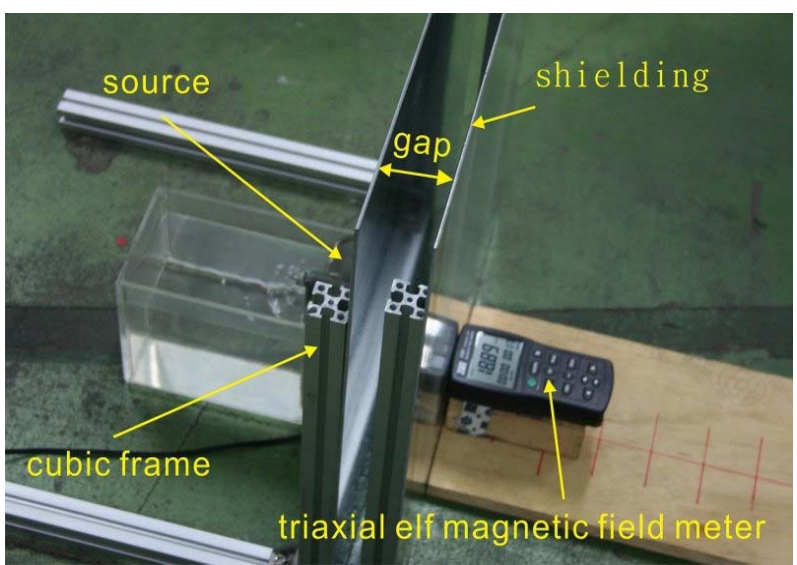

(b)

Figure 4. Graphical descriptions for measuring the decay rate and the transmission ratio with various materials as well as different plate gaps. (a) Schema of the experimental setup; (b) Photo of the experimental setup.

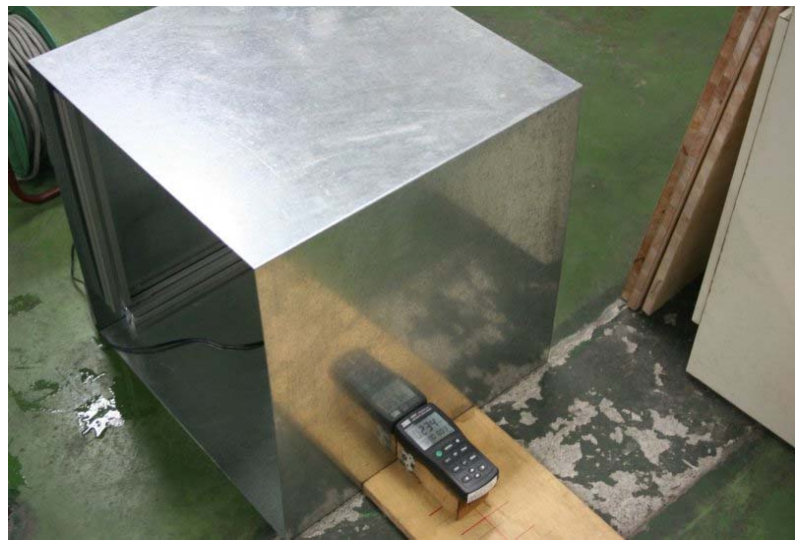

Figure 5. Photo of the experimental setup for measuring the magnetic field loop effect.

Figure 9 shows relationship between the transmission ratio and the measured distances using the iron plates. The applied plate thicknesses are 1,2, and $5 \mathrm{~mm}$. The result shows that the transmission ratio is proportional to the measured distance. In comparison with results of the 


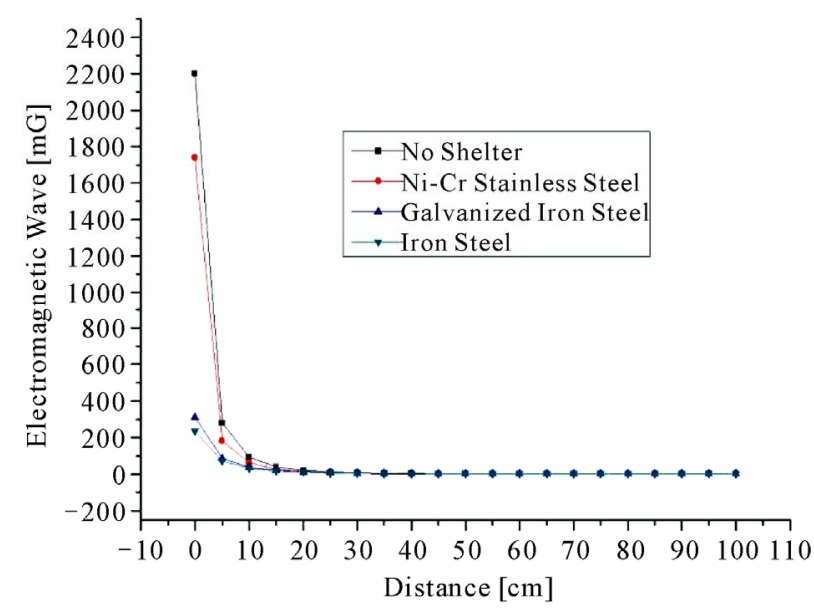

Figure 6. Relationship between the penetrated magnetic field intensity and the measured distances with various shielding materials, where all plate thicknesses are $1 \mathrm{~mm}$.

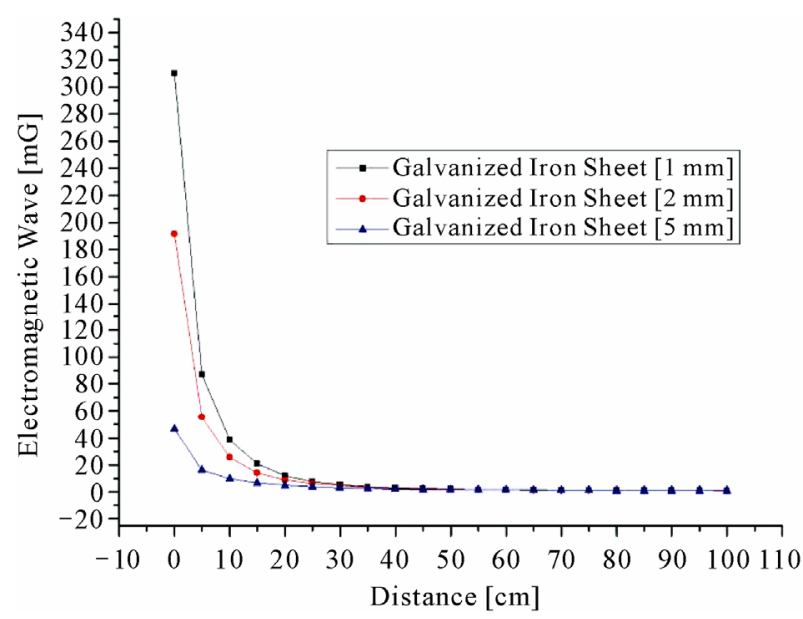

Figure 7. The relationship between penetrated magnetic field intensity and the measured distances with the different plate thicknesses of the iron.

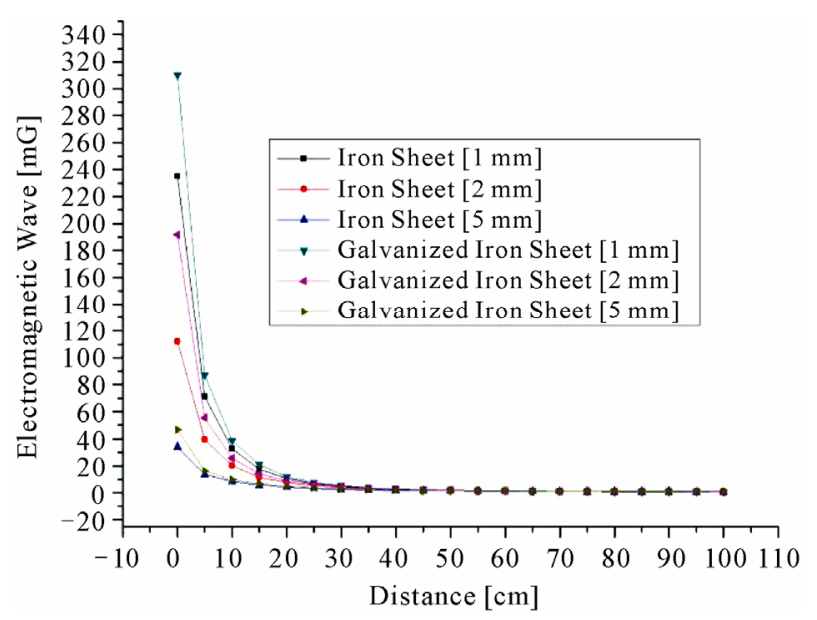

Figure 8. Comparison of the galvanized iron and the iron protection for magnetic field.

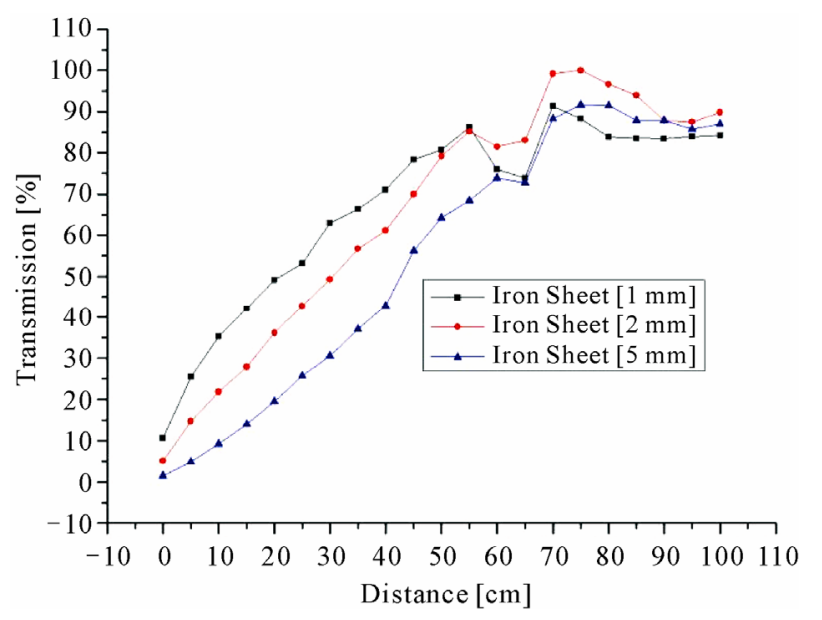

Figure 9. Relationship between the transmission ratio and the measured distances with the iron plates.

decay rate in Figure $\mathbf{7}$ indicates the good agreement. The magnetic field intensity strongly decays nearby the emission source. The magnetic field intensity far away from the emission source shows almost no variation.

The measurements using two plates with a gap is to consider influence of the gap. Figure $\mathbf{1 0}$ shows relationship between the penetrated magnetic field intensity and the measured distances using the $1 \mathrm{~mm}$ thickness iron plates with the 0,3 , and $5 \mathrm{~cm}$ gap sizes. The shielding plates with gap have better protection than the same thickness plate, but it is independent on the gap size.

Figure 11 shows relationship between the penetrated magnetic field intensity and the measured distances with the shielding plate nearby the emission source in comparison with the shielding plate nearby the measuring meter. The result shows that protection with the shielding plate nearby the emission source is better than the shielding plate nearby the measuring meter. That is, to protect the electromagnetic field using the shielding plate should be built as close as the emission source.

Figure 12 shows relationship between penetrated magnetic field intensity and the measured distances with the closed loop circle squared galvanized iron plate. The result shows that the alternating magnetic field yields no loop effect in the shielding material.

Summarizing the results indicates that iron plate has better shielding effect due to it has smaller skin depth [11]. The thicker the shielding plate, the fewer the penetrated intensity of the electromagnetic wave is. Shielding plate close to the emission source receives better shielding effect due to the near hyperbolic damping curve of the electromagnetic wave intensity vs. the skin depth as shown in Section 2 [11]. In other words, the closer the shielding plate to the emission source, the larger the shielded ratio of the electromagnetic wave. Moreover, applying loop structure of the shielding material has not 


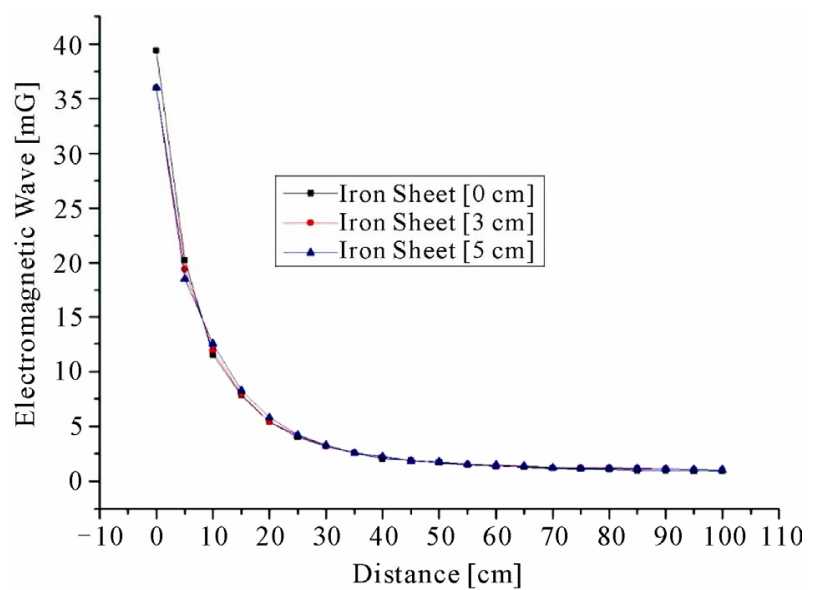

Figure 10. The relationship between penetrated magnetic field intensity and the measured distances using the $1 \mathrm{~mm}$ thickness iron plates with the 0,3 , and $5 \mathrm{~cm}$ gap sizes.

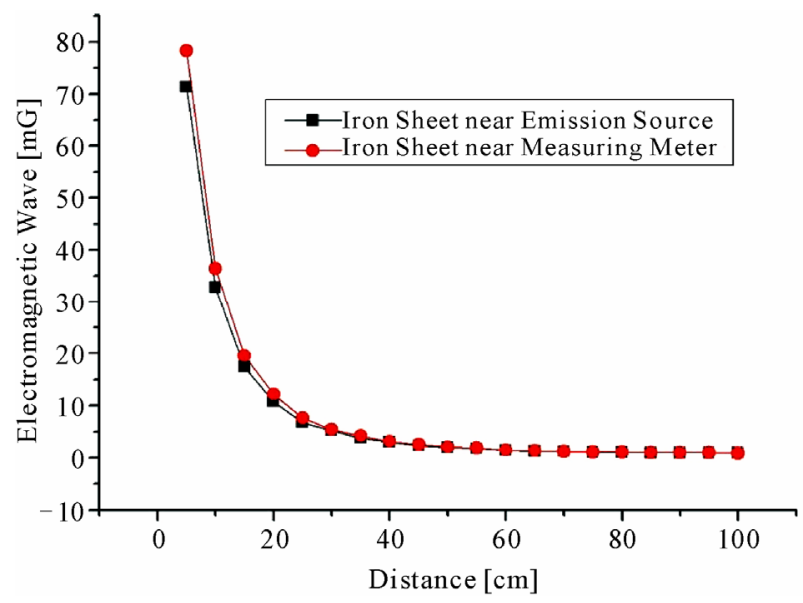

Figure 11. Relationship between the penetrated magnetic field intensity and the measured distances with the shielding plate nearby the emission source.

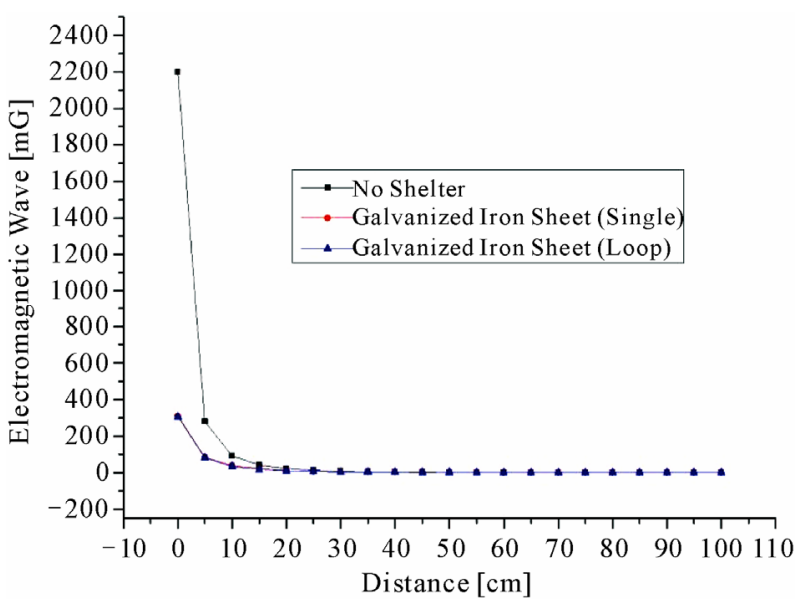

Figure 12. The relationship between penetrated magnetic field intensity and the measured distances with the closed loop circle square galvanized iron plate. helped the shielding effect. The reason is that the generated current in the shielding material is not enough to cause the loop current.

\section{Conclusions}

Measuring influence of the material, the shielding plate thickness, the shielding plate gap, and the loop effect on the decay rate and the transmission ratio of electromagnetic field by the shielding protection are successfully carried out. The results show that the iron $(\mathrm{Fe})$ has the best protection for the magnetic field. The larger amount iron constituent and the thicker shielding plate, the better shielding effect for the magnetic field makes. The transmission ratio is proportional to distance between the emission source and the measuring meter. Two plates with gap is better than the same sum thick plate for shielding effect, but it is independent on the gap size. The shielding plate close to the emission source has better protection.

\section{Acknowledgements}

The authors would like to acknowledge the financial support from the National Taipei University of Technology.

\section{References}

[1] D. K. Cheng, "Field and Wave Electromagnetics," Addison-Wesley Publishing Co., Boston, Jwang Yuan Publishing Co., Taipei, 1988.

[2] M. A. S. Oviedo, O. A. Araujo, R. Faez, M. C. Rezende and M. D. Paoli, "Antistatic Coating and Electromagnetic Shielding Properties of a Hybrid Material Based on Polyaniline/Organoclay Nanocomposite and EPDM Rubber," Synthetic Metals, Vol. 156, No. 18-20, 2006, pp. 1249-1255. doi:10.1016/i.synthmet.2006.09.003

[3] M. R. Hernand and G. G. Karady, "Attenuation of Low Frequency Magnetic Fields Using Active Shielding," Electric Power Systems Research, Vol. 45, No. 1, 1998, pp. 57-63. doi:10.1016/S0378-7796(97)01232-7

[4] E. Hakansson, A. Amiet, S. Nahavandi and A. Kaynak, "Electromagnetic Interference Shielding and Radiation Absorption in Thin Polypyrrole Films," European Polymer Journal, Vol. 43, No. 1, 2007, pp. 205-213. doi:10.1016/j.eurpolymj.2006.10.001

[5] D. D. L. Chung, "Electromagnetic Interference Shielding Effectiveness of Carbon Materials," Carbon, Vol. 39, No. 2, 2001, pp. 279-285. doi:10.1016/S0008-6223(00)00184-6

[6] K. K. S. Kumar, S. Geetha and D. C. Trivedi, "Freestanding Conducting Polyaniline Film for the Control of Electromagnetic Radiations," Current Applied Physics, Vol. 5, No. 6, 2005, pp. 603-608. 
doi:10.1016/j.cap.2004.08.004

[7] S. Keeping and G. Zhenyn, "Laboratory Research on Shielding Effect of a Novel Conductive Cloth to Microwave Radiation," Journal of Electrostatics, Vol. 252, 1998, pp. 125-129. doi:10.1016/S0304-3886(98)00032-1

[8] H. C. Lee, J. Y. Kim, C. H. Noh, K. Y. Song and S. H. Cho, "Selective Metal Pattern Formation and Its EMI Shielding Efficiency," Applied Surface Science, Vol. 252, No. 8, 2006, pp. 2665-2672. doi:10.1016/j.apsusc.2005.03.206

[9] J. L. Huang, B. S. Yau, C. Y. Chen, W. T. Lo and D. F. Lii, "The Electromagnetic Shielding Effectiveness of In- dium tin Oxide Films," Ceramics International, Vol. 27, No. 3, 2001, pp. 363-365.

doi:10.1016/S0272-8842(00)00088-2

[10] Z. Dou, G. Wu, X. Huang, D. Sun and L. Jiang, "Electromagnetic Shielding Effectiveness of Aluminum Alloy-Fly Ash Composites," Composites: Part A, Vol. 38, 2007, pp. 186-191. doi:10.1016/j.compositesa.2006.01.015

[11] P. Lorrain and D. R. Corson, "Electromagnetic Fields and Waves," W. H. Freeman and Company, New York, 1987.

[12] E. Hecht, "Optics," Addison-Wesley Publishing Company, Boston, 2002. 